\title{
Upscaling of the single-phase flow and heat transport in fractured geothermal reservoirs using nonlocal multicontinuum method
}

\author{
Maria Vasilyeva $^{1,2} \cdot$ Masoud Babaei $^{3}$ (D) Eric T. Chung ${ }^{4}$. Valentin Alekseev ${ }^{2}$
}

Received: 31 August 2018 / Accepted: 22 March 2019 / Published online: 6 April 2019

(C) The Author(s) 2019

\begin{abstract}
In this work, we consider a single-phase flow and heat transfer problem in fractured geothermal reservoirs. Mixed dimensional problems are considered, where the temperature and pressure equations are solved for porous matrix and fracture networks with transfer term between them. For the fine-grid approximation, a finite volume method with embedded fracture model is employed. To reduce size of the fine-grid system, an upscaled coarse-grid model is constructed using the nonlocal multicontinuum (NLMC) method. We present numerical results for two-dimensional problems with complex fracture distributions and investigate an accuracy of the proposed method. The simulations using upscaled model provide very accurate solutions with significant reduction in the dimension of problem.
\end{abstract}

Keywords Geothermal heat recovery $\cdot$ Multiscale modeling $\cdot$ Fractured media $\cdot$ Nonlocal multicontinuum method

\section{Introduction}

An abundant source of geothermal energy that can be found almost everywhere deep beneath the earth's surface, hot dry rock (HDR), can offer sustainable energy to reduce dependence on fossil fuels. These heat sources, however, are contained within hot but essentially dry, impervious crystalline basement rocks that are not naturally fractured [22]. As such, there are numerous challenges in producing heat from HDR. Firstly, the exploitable resources occur at depths of more than $2 \mathrm{~km}$. Secondly, to recover heat from such systems, unlike hot sedimentary aquifers with sufficiently large permeabilities [4, 14, 57], operations are needed to develop permeable and connected fractures

Masoud Babaei

masoud.babaei@manchester.ac.uk

1 Texas A\&M University, College Station, TX, USA

2 North Eastern Federal University, Yakutsk, Russia

3 School of Chemical Engineering and Analytical Science, University of Manchester, Manchester, UK

4 Department of Mathematics, The Chinese University of Hong Kong, Shatin, Hong Kong first [38]. Finally, mining heat from such rocks is by introducing cold water into the hot rocks to be heated, and then producing hot water or steam in doublet well configurations. The resultant geothermal system is referred to as Enhanced Geothermal Systems (EGS). The potential of EGS as a future means of providing green energy has been suggested by number of authors [19]. However, the main technical difficulty has been pointed out to stem from the challenges in prediction of fully coupled thermal-hydromechanical processes during and after creation of fractures and permeable conduits.

In order to efficiently produce from HDR, a good understanding of coupled processes through created networks of fractures are critically needed. This understanding can be achieved through the employment of powerful computational resources to set up and solve computationally demanding models with sufficient temporal and spatial resolutions. However, due to the uncertain nature of subsurface systems, a deterministic model of EGS-induced fracture networks cannot be constructed and multiple realizations and stochastic modeling are needed. This means that the computations are multiplied by a number of realizations, thereby leading to even more excessive computations. To this end, it has now been a decades-long research objective to develop efficient computational methods to solve coupled thermo- 
hydraulic, as well as chemical-hydraulic processes in subsurface systems. In the following paragraphs, we briefly review the background and literature of multiscale methods for fractured geothermal media and EGS applications.

Fracture networks have complex geometries. Fractures have very small thickness compared to typical reservoir sizes and have a significant impact on the flow processes. A common approach to model fracture media is to consider discrete fractures as lower dimensional objects [15, 20, 34]. This results in a coupled mixed dimensional mathematical models, where we have a $d$ - dimensional equation for flow in porous matrix and a $(d-1)$-dimensional equation for fracture networks. The cross-flow equilibrium between the flow in fracture and matrix is described by transfer terms.

\subsection{Fine-grid approximation}

Whereas in reservoir simulations the finite volume method is widely used, for numerical simulation of the fractured porous media various numerical approaches have been developed that can be classified by the types of meshing techniques. For the unstructured meshes where fractures are gridded explicitly, the discrete fracture models are used. In another approach, called the embedded fracture model (EFM) [21, 24, 45], the fractures are not resolved by grid but are considered as overlaying continua. In EFM, matrix and fracture are viewed as two porosity types co-existing at the same spatial location, thus simple structured meshes can be used for the domain discretization. The transfer term between matrix and fracture appears as an additional source or sink term and is assumed to exist in entire simulation domain. This concept can be classified in the class of dual-continuum or multicontinuum models $[3,5,23,55]$.

\subsection{Multiscale methods}

The fine-grid simulation of the processes in fractured porous media is very expensive for complex fracture distribution, where accuracy depends on honoring high-resolution discrete fractures. In general subsurface problems, to reduce the dimension of fine-scale system, multiscale methods or upscaling techniques are used [16, 27, 28, 33, 56]. Also recently, the multiscale methods have been adapted for geothermal and EGS problems [25, 26, 29, 30, 35, 39, 43].

Vasilyeva et al. [48] presented the multiscale model reduction techniques based on the generalized multiscale finite element method (GMsFEM) for geothermal fractured reservoir simulation. The general idea of GMsFEM is to first solve some local problems to get snapshot spaces, then design suitable spectral problems to obtain important modes which can be used to construct multiscale basis $[9,10,17]$.
The resulting multiscale space contains basis functions that take into account the subgrid-scale heterogeneity. The multiscale scale solution found in this space provides an accurate approximation of flow and transport $[1,2,11,18]$.

Nissen et al. [35] presented an upscaling methodology for geothermal heat transport in fractured porous media, where upscaling is based on different strategies for advective and conductive terms. The coarse-scale advective term is constructed from sums of fine-scale fluxes, whereas the coarse-scale conductive term is constructed based on numerically computed basis functions. Praditia et al. [39] obtained a nonlinear time-dependent (transient) multiscale coarse-scale system for both pressure and temperature unknowns based on locally solved elliptic basis functions.

\subsection{This work}

In contrast to the above-mentioned techniques, in this work, we develop an accurate upscaled coarse-grid model for single-phase fluid flow and heat transfer problems in fractured porous media. We use the recently developed nonlocal multicontinuum (NLMC) upscaling method [13, $49,50]$. In this paper, NLMC is expanded to geothermal systems as well where there are coupled flow and heat transport. The method is originally developed for Darcy flow in fractured media without heat transfer. The main idea is to use one macroscopic variable for each fracture network and the matrix within each coarse region (hence multicontinuum). This is very important for fractures as one single macroscopic variable for each coarse region is not enough to give reasonable accuracy. For example, the flow within each fracture network or matrix can move with highly disparate velocities. Another important motivation of NLMC method is localization. It is clear that the fractures can give long-range global effects. By using the proposed energy minimization principle, the resulting basis functions have exponential decay away from an oversampled region of a target coarse element. This allows a systematic coupling of nonlocal macroscopic variables and therefore enhances the accuracy of the method.

Construction of the upscaled model is based on the solution of local elliptic problems for flow, and on the local convection-diffusion problem for heat transport. The multiscale basis functions for transport problem account for the fine-grid flow characteristics in both matrix and fractures media that lead to more accurate coarse-grid approximation. Compared to Nissen et al. [35], Praditia et al. [39], and Vasilyeva et al. [48] where local elliptic problems are solved to construct transport multiscale basis functions, NLMC provides coarse-grid approximation whose degrees of freedoms have physical meanings. As a result, we have 
an accurate general multiscale framework for both flow and transport problems in fractured porous media, where we model fractures embedded into the coarse mesh cell. The upscaled model has only one additional coarse degree of freedom for each fracture network and is directly related to the well-known dual-continuum approaches, which have been commonly used in approximating subgrid effects for flow and transport in fractured media.

\subsection{Outline}

This paper is organized as follows. In Section 2, we consider mathematical model for coupled fluid flow and heat transfer in fractured porous media. Next in Section 3, we present finite volume fine-grid approximation with the embedded fracture model. In Section 4, we propose an upscaled coarse-grid model for flow and transport in fractured porous media. We present numerical examples for two model geometries in Section 5, and show that the proposed method can achieve a good accuracy with a very few degrees of freedom (DoF). This is followed by Section 6 in which conclusions and future works are presented.

\section{Problem formulation}

We consider single-phase coupled fluid flow and heat transfer in fractured porous media with highly porous and permeable fractures criss-crossing an otherwise homogeneous matrix continuum (Fig. 1).

Let $\Omega \subset \mathcal{R}^{2}$ be the computational domain for the porous matrix and $\gamma \subset \mathcal{R}^{1}$ be the lower dimensional domain for fractures. The mathematical model of the coupled process is described by the coupled mixed dimensional system of equations for temperature and pressure in the porous matrix in $\Omega$ and in the fracture network in $\gamma$. The change of density

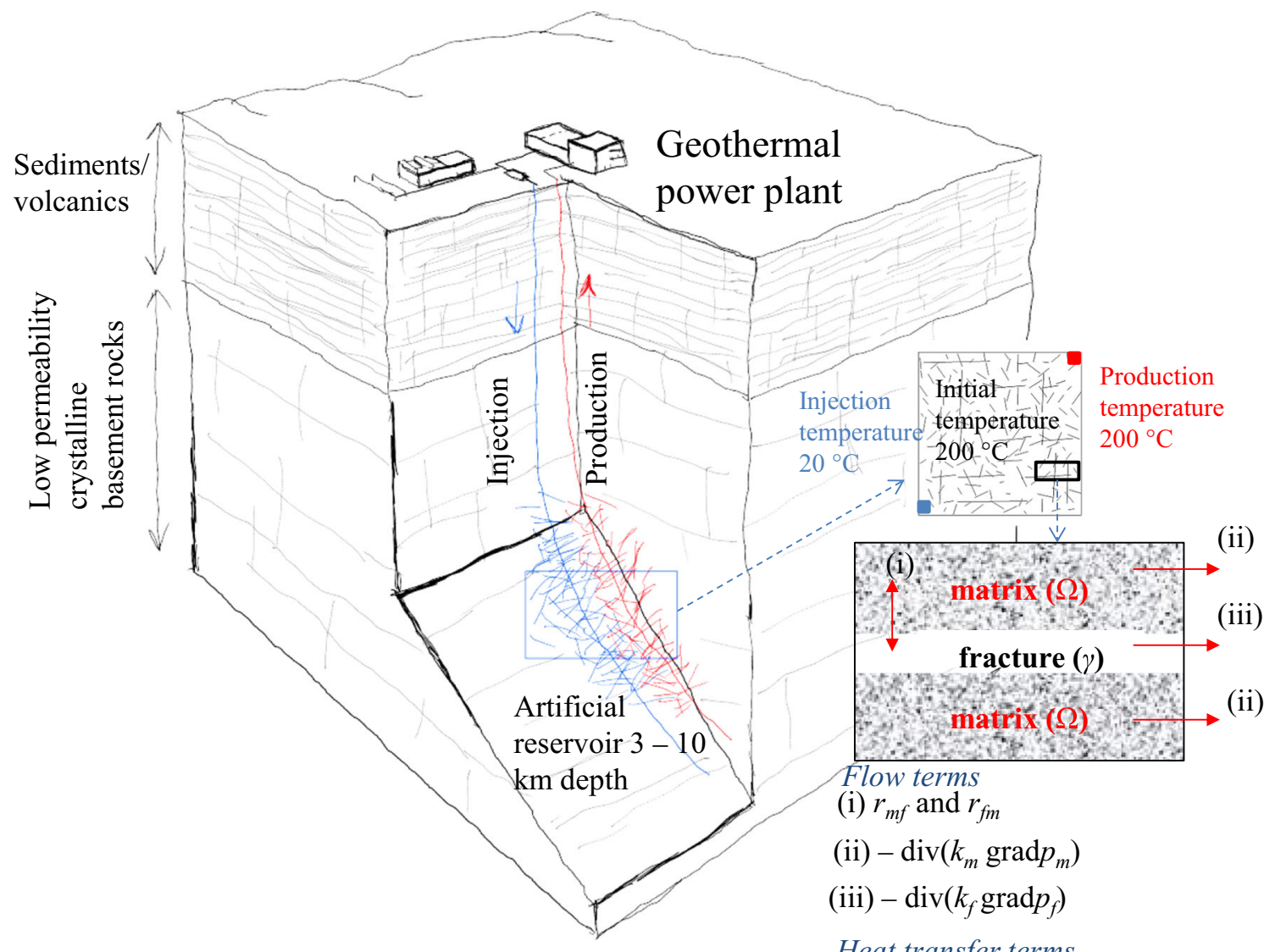

Heat transfer terms

(i) $L_{m f}$ and $L_{f m}$

(ii) $(c \rho)_{m} \operatorname{div}\left(q_{m} T_{m}\right)-\operatorname{div}\left(\lambda_{m} \operatorname{grad} p_{m}\right)$

(iii) $(c \rho)_{f} \operatorname{div}\left(q_{f} T_{f}\right)-\operatorname{div}\left(\lambda_{f} \operatorname{grad} p_{f}\right)$

Fig. 1 A schematic of EGS doublet systems, a representation of the study area, and fracture-matrix flow and heat transfer terms of Eqs. 2 and 3. The figure is modified from Tester et al. [47] and Vasilyeva et al. [48] 
$\rho$ in both matrix and fracture media is related to the changes in temperature and pressure

$\rho(p, T)=\rho_{0}\left(1-c_{T}\left(T-T_{0}\right)+c_{p}\left(p-p_{0}\right)\right)$,

where $c_{T}$ is the fluid thermal expansion coefficient $\left[1 /{ }^{\circ} \mathrm{C}\right]$, and $c_{p}[1 / \mathrm{Pa}]$ is the coefficient of fluid isothermal compressibility [44].

For fluid flow in the porous matrix and fracture networks, we have the following system of equations

$\begin{array}{ll}a_{m} \frac{\partial p_{m}}{\partial t}-s_{m} \frac{\partial T_{m}}{\partial t}-\operatorname{div}\left(k_{m} \operatorname{grad} p_{m}\right)+r_{m f}=g_{m}^{p}, & x \in \Omega, \\ a_{f} \frac{\partial p_{f}}{\partial t}-s_{f} \frac{\partial T_{f}}{\partial t}-\operatorname{div}\left(k_{f} \operatorname{grad} p_{f}\right)-r_{f m}=g_{f}^{p}, & x \in \gamma,\end{array}$

where

$k_{m}=\frac{\kappa_{m}}{\mu}, \quad k_{f}=\frac{\kappa_{f}}{\mu}, \quad a_{m}=\phi c_{p}, \quad a_{f}=c_{p}, \quad s_{m}=\phi c_{T}, \quad s_{f}=c_{T}$

and

$r_{m f}=\sigma \eta_{m}\left(p_{m}-p_{f}\right), \quad r_{f m}=\sigma \eta_{f}\left(p_{m}-p_{f}\right)$.

where $\phi$ is the porosity of matrix (assumed constant), fractures are highly porous $\left(\phi_{f}=1\right)$ and assumed planar, $p_{m}$ and $p_{f}$ are the matrix and fracture pressures, $q_{m}$ and $q_{f}$ are Darcy velocities in matrix and fractures, $\kappa_{m}$ and $\kappa_{f}$ are the matrix and fracture permeability, $\mu$ is the fluid viscosity, $\sigma \approx C I \frac{\kappa_{m}}{\mu}$ with $\eta_{m}=1 / V_{m}, \eta_{f}=1 /\left(h A_{f}\right)$, where $V_{m}$ is the volume of matrix of grididblock, $A_{f}$ is the fracture interface with in a matrix gridblock, and $h$ is the thickness of gridblock. Also, $C I$ is the gridblock-specific connectivity index (with the unit of $\mathrm{m}$ ) that is calculated similar to the method defined by Hajibeygi et al. [24]. Finally in Eq. $2, g_{m}^{p}$ and $g_{m}^{p}$ refer to pressure source and sink terms contributing to the pressure variation in the matrix and fracture, respectively. They have a unit of $[1 / s]$ as they refer to source/sink mass rate $\partial \rho / \partial t$ divided by $\rho_{0}$.

For heat transfer in fractured porous media, we have the following equations

$$
\begin{aligned}
(c \rho)_{m} \frac{\partial T_{m}}{\partial t} & +(c \rho)_{w} \operatorname{div}\left(q_{m} T_{m}\right) \\
& -\operatorname{div}\left(\lambda_{m} \operatorname{grad} T_{m}\right)+L_{m f}=(c \rho)_{w} g_{m}^{T}, \quad x \in \Omega, \\
(c \rho)_{f} \frac{\partial T_{f}}{\partial t} & +(c \rho)_{w} \operatorname{div}\left(q_{f} T_{f}\right) \\
& -\operatorname{div}\left(\lambda_{f} \operatorname{grad} T_{f}\right)-L_{f m}=(c \rho)_{w} g_{f}^{T}, \quad x \in \gamma,
\end{aligned}
$$

where $q_{m}$ and $q_{f}$ are the fluid velocity in the porous matrix and fracture network, respectively

$q_{m}=-k_{m} \operatorname{grad} p_{m} \quad$ and $\quad q_{f}=-k_{f} \operatorname{grad} p_{f}$.

For porous matrix and fractures, we have also the following relations

$(c \rho)_{m}=(1-\phi) c_{r} \rho_{r}+\phi c_{w} \rho_{w}, \quad(c \rho)_{f}=c_{w} \rho_{w}$,

$\lambda_{m}=(1-\phi) \lambda_{r}+\phi \lambda_{w}, \quad \lambda_{f}=\lambda_{w}$, where $\rho_{r}, c_{r}, \rho_{w}, c_{w}$ denote density and specific heat of the solid and fluid phases, respectively, and $\lambda_{r}$ and $\lambda_{w}$ are the thermal conductivity of the solid and fluid phases, respectively [37, 40, 53, 54]. Underlying assumptions in Eq. 3 are that: (a) the volumetric heat capacity $(c \rho)_{m}$ and $(c \rho)_{f}$ are constant and can be taken out outside the partial derivatives, and (b) the differential internal energy can be written as $d U=c \rho d T$. Furthermore, in Eq. 3, $g_{m}^{T}=g_{m}^{p} T_{m}$ and $g_{f}^{T}=g_{f}^{p} T_{f}\left[^{\circ}\right] \mathrm{C} / \mathrm{s}$ refer to temperature source and sink terms contributing to the temperature variation in the matrix and fracture, respectively.

For heat transfer between matrix and fractures, we have

$L_{m f}=\beta \eta_{m}\left(T_{m}-T_{f}\right)+(c \rho)_{w} \sigma \eta_{m}\left(p_{m}-p_{f}\right) T_{m f}$,

$L_{f m}=\beta \eta_{f}\left(T_{m}-T_{f}\right)+(c \rho)_{w} \sigma \eta_{f}\left(p_{m}-p_{f}\right) T_{m f}$,

where $\beta \approx C I \lambda_{m}$ and in the upwind scheme, we set $T_{m f}=T_{m}$ if $r_{m f}>0$ and $T_{m f}=T_{f}$ else [39]. The first term is responsible for conductive heat transfer between fracture and matrix, while the second term is responsible for convective (pressure difference driven) heat transfer between fracture and matrix [39].

\section{Fine-grid approximation}

In this section, we present the fine-grid approximations of the above models. Based on these fine-grid methods, we will then in the next section develop our new upscaling schemes. For the fine-grid approximations, we will use the finite volume method that is widely used for reservoir simulations. Specifically, we employ the cell-centered finite-volume method with two-point flux approximation together with the use of the embedded fracture model [8, 24, 45, 46].

Let $\mathcal{T}_{h}=\cup_{i} K_{i}$ be a structured fine grid with rectangular cells of the domain $\Omega$ and $\mathcal{E}_{\gamma}=\cup_{l} \gamma_{l}$ be the fracture mesh. We let $N_{f}^{m}$ be the number of cells in $\mathcal{T}_{h}$ and $N_{f}^{f}$ be the number of cells related to fracture mesh $\mathcal{E}_{\gamma}$ (Fig. 2). Using the embedded fracture model, we have the following discrete systems for the heat transfer equation and the flow equation:

- Fluid flow:

$$
\begin{aligned}
& a_{m, i} \frac{p_{m, i}-\check{p}_{m, i}}{\tau}\left|\xi_{i}\right|-s_{m, i} \frac{T_{m, i}-\check{T}_{m, i}}{\tau}\left|\xi_{i}\right| \\
& \quad+\sum_{j}\left(q_{m} \cdot \mathbf{n}\right)_{i j}+\sigma_{i l}\left(p_{m, i}-p_{f, l}\right)=g_{m, i}^{p}\left|\xi_{i}\right|, \quad \forall i=1, N_{f}^{m} \\
& a_{f, l} \frac{p_{f, l}-\check{p}_{f, l}}{\tau}\left|\iota_{l}\right|-s_{f, l} \frac{T_{f, l}-\check{T}_{f, l}}{\tau}\left|\iota_{l}\right| \\
& \quad+\sum_{n}\left(q_{f} \cdot \mathbf{n}\right)_{l n}-\sigma_{i l}\left(p_{m, l}-p_{f, i}\right)=g_{f, l}^{p}\left|\iota_{l}\right|, \quad \forall l=1, N_{f}^{f}
\end{aligned}
$$


- Heat transfer:

$$
\begin{aligned}
&(c \rho)_{m, i} \frac{T_{m, i}-\check{T}_{m, i}}{\tau}\left|\xi_{i}\right|+(c \rho)_{w, i} \sum_{j}\left(q_{m} \cdot \mathbf{n}\right)_{i j} T_{m, i j}+(c \rho)_{w, i} T_{m f, i l} \sigma_{i l}\left(p_{m, i}-p_{f, l}\right) \\
&+\sum_{j} D_{i j}^{m}\left(T_{m, i}-T_{m, j}\right)+\beta_{i l}\left(T_{m, i}-T_{f, l}\right)=(c \rho)_{w, i} g_{m, i}^{T}\left|\xi_{i}\right|, \quad \forall i=1, N_{f}^{m} \\
&(c \rho)_{f, l} \frac{T_{f, l}-\check{T}_{f, l}}{\tau}\left|\iota_{l}\right|+(c \rho)_{w, l} \sum_{n}\left(q_{f} \cdot \mathbf{n}\right)_{l n} T_{f, l n}-(c \rho)_{w, i} T_{m f, i l} \sigma_{i l}\left(p_{m, i}-p_{f, l}\right) \\
& \quad+\sum_{n} D_{l n}^{f}\left(T_{m, l}-T_{m, n}\right)-\beta_{i l}\left(T_{m, i}-T_{f, l}\right)=(c \rho)_{w, l} g_{f, l}^{T}\left|\iota_{l}\right|, \quad \forall l=1, N_{f}^{f} .
\end{aligned}
$$

In the above systems,

$\left(q_{m} \cdot \mathbf{n}\right)_{i j}=W_{i j}^{m}\left(p_{m, i}-p_{m, j}\right), \quad\left(q_{f} \cdot \mathbf{n}\right)_{l n}=W_{l n}^{f}\left(p_{f, l}-p_{f, n}\right)$, where $\mathbf{n}$ is the normal vector, $W_{i j}^{m}=k_{m}\left|E_{i j}\right| / d_{i j}\left(\left|E_{i j}\right|\right.$ is the length of facet between cells $\xi_{i}$ and $\xi_{j}, d_{i j}$ is the distance between midpoint of cells $\xi_{i}$ and $\xi_{j}$ ), and $W_{l n}^{f}=k_{f} / \Delta_{l n}$ ( $\Delta_{l n}$ is the distance between points $l$ and $n$ ). Similarly, for the heat equation, we have $D_{i j}^{m}=\lambda_{m}\left|E_{i j}\right| / d_{i j}$ and $D_{l n}^{f}=\lambda_{f} / \Delta_{l n}$. We set $\sigma_{i l}=C I_{i l} k_{m}$ and $\beta_{i l}=C I_{i l} \lambda_{m}$, if $\gamma_{l} \subset K_{i}$ and zero else, where we take $\eta_{m}=1 /\left|\xi_{i}\right|$ and $\eta_{f}=1 /\left(h\left|\iota_{l}\right|\right)$. As previously mentioned for transfer terms between matrix and fracture, the connectivity index is $C I_{i l}=A_{i l}^{f m} /<d_{i l}^{f m}>$, where $A_{i l}^{f m}$ is the area fraction of fracture element $\iota_{l}$ with the matrix element $\xi_{i}$, and $d_{i l}^{f m}$ is the average distance of the fracture and matrix elements [24]. For the time discretization, we use the standard backward Euler implicit scheme, where $\check{T}_{m}, \check{T}_{f}, \check{p}_{m}, \check{p}_{f}$ are the solutions from previous time step and $\tau$ is the given time step size.

We split the coupled system of pressure and temperature (Eqs. 4 and 5), by first assuming that we know temperature in the pressure equation, and then solve the temperature equation. The size of fine-grid system is $\mathrm{DoF}_{f}=N_{f}^{m}+N_{f}^{f}$. Details of fine-scale solution are given in the Appendix.

\section{Coarse-grid nonlocal multicontinuum upscaling}

We derive an upscaling technique based on the nonlocal multicontinuum method for the following general fine-scale problem:

$M \frac{u-\check{u}}{\tau}+A u=F$,

where

$A=\left(\begin{array}{cc}A_{m} & A_{m f} \\ A_{f m} & A_{f}\end{array}\right), \quad M=\left(\begin{array}{cc}M_{m} & 0 \\ 0 & M_{f}\end{array}\right), \quad F=\left(\begin{array}{l}F_{m} \\ F_{f}\end{array}\right)$. and $u=\left(u_{m}, u_{f}\right)^{T}$. We remark that the system of Eq. 6 is motivated by the fine-scale systems detailed in the Appendix). In the above system of Eq. 6, $u_{m}$ denotes the solution on the matrix part and $u_{f}$ denotes the solution on the fractures in the current time step. Moreover, $\tau>0$ represents the time step size, and $\check{u}$ represents the solution in the previous time step. The matrices $M, A$ and the source $F$ are defined for the pressure equation and the temperature equation as follows:

- For the pressure equation, $u=p$ :

$$
M=M^{\text {flow }}, \quad F=G^{\text {flow }}+S^{\text {flow }} \frac{T-\check{T}}{\tau}, \quad A=A^{\text {flow }} .
$$

- For the temperature equation, $u=T$ :

$$
M=M^{\text {heat }}, \quad F=G^{\text {heat }}+S^{\text {heat }} \frac{p-\check{p}}{\tau}, \quad A=A^{\text {heat }},
$$

where we remark that $A^{\text {heat }}$ contains both the diffusive and convective parts.

The main idea of the NLMC method is the use of multiple upscaled variables per coarse element and local multiscale basis functions to form the upscaled system [13, 49-51]. First of all, for each coarse element, the upscaled variables are the average values of the solution on separate fracture networks as well as the matrix part. This leads to a multicontinuum approach. Next, for each continuum within a coarse element, we solve a constrained local problem on an oversampling domain, which is obtained by enlarging the coarse element by a few coarse-grid layers. More precisely, we solve the given PDE on an oversampled region subject to the conditions that the solution has mean value zero on all continua except the one that is specified. Based on the mathematical foundation in Chung et al. [12], this localized computation is motivated on an exponential decay property. Namely, the solution of this constrained problem has exponential decay outside an oversampled region, even for the case when the domain contains long fractures. Moreover, the local basis functions defined in this way give 
Fig. 2 Illustration of a 2D fine grid, $\mathcal{T}_{h}$ and $\mathcal{E}_{\gamma}$

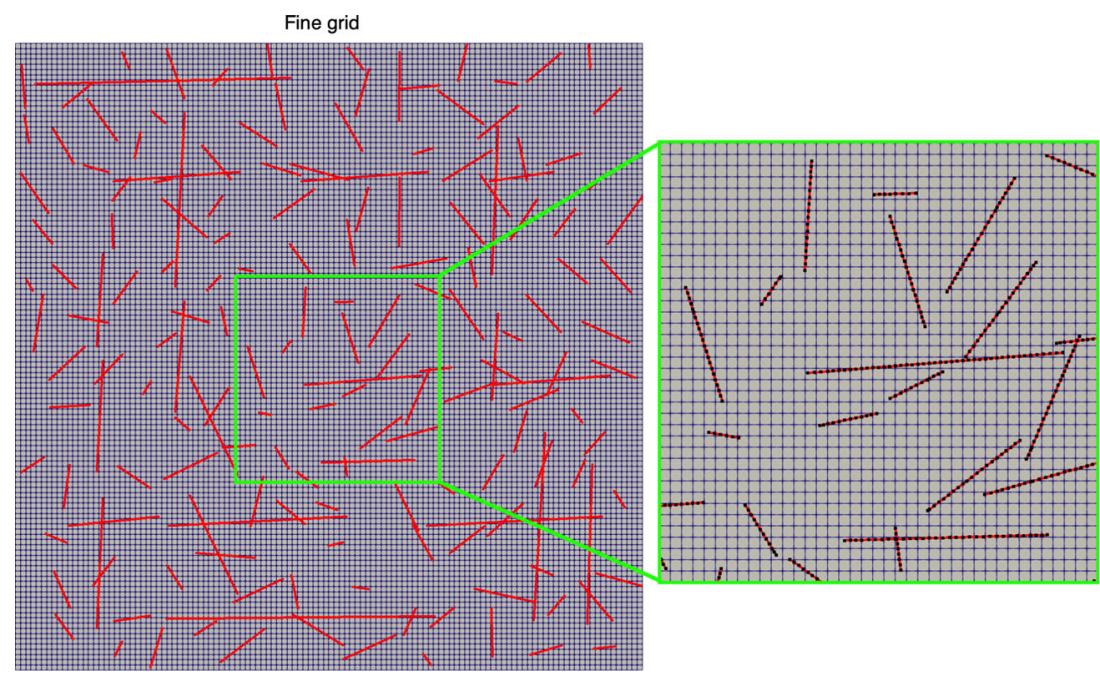

a systematic coupling of the upscaled variables in a nonlocal way. This is crucial for our NLMC method for fractured media as the solution typically contains nonlocal and long range effects.

Next, we define some notations. Let $\mathcal{T}^{H}$ be a coarse partition of the domain $\Omega, K_{i} \in \mathcal{T}^{H}$ be the $i$-th coarse block $\left(i=1,2, \ldots, N_{c}\right.$, where $N_{c}$ is the number of coarse grid cells), and let $K_{i}^{+}$be the corresponding oversampled region obtained by expanding the coarse block $K_{i}$ by several coarse-grid layers. We denote the fractures by $\gamma$. We write $\gamma=\cup_{l=1}^{L} \gamma^{(l)}$, where $\gamma^{(l)}$ denotes the $l$-th fracture network and $L$ is the total number of fracture networks. In addition, we write $\gamma_{j}^{(l)}=K_{j} \cap \gamma^{(l)}$ to be the fracture inside coarse cell $K_{j}$ and $L_{j}$ is the number of fractures in $K_{j}$. For a given coarse element $K_{i}$ and a given continuum (either the matrix part in $K_{i}$ or a fracture network $\gamma_{i}^{(l)}$, we will solve a local problem in the oversampled domain $K_{i}^{+}$ with some constraints (later explained) to find a multiscale basis function $\psi_{l}^{i}$ corresponding to this coarse element and this continuum. We remark that this basis function has exponential decay outside $K_{i}^{+}$.

We construct a set of local multiscale basis functions $\psi_{0}^{i}$ and $\psi_{l}^{i}\left(l=1,2, \cdots, L_{i}, L_{i}\right.$ is the number of fracture networks in $K_{i}$ ) by solving a local problem on an oversampled region. Here, we use the subscript zero to represent the matrix part. The basis functions are required to satisfy some constraints in each coarse element $K_{j} \subset K_{i}^{+}$. These constraints are described as follows.

For the matrix part, we require the basis function $\psi_{0}^{i}$ to satisfy the following on each $K_{j} \subset K_{i}^{+}$:

$$
\int_{K_{j}} \psi_{0}^{i} d x=\delta_{i, j}, \quad \int_{\gamma_{j}^{(l)}} \psi_{0}^{i} d s=0, \quad l=\overline{1, L_{j}}
$$

For the $n$-th fracture network in $K_{i}$, we require the basis function $\psi_{n}^{i}$ to satisfy the following on each $K_{j} \subset K_{i}^{+}$:

$\int_{K_{j}} \psi_{n}^{i} d x=0, \quad \int_{\gamma_{j}^{(l)}} \psi_{n}^{i} d s=\delta_{i, j} \delta_{n, l}, \quad l=\overline{1, L_{j}}$

We remark that for a fixed coarse element $K_{i}$ and a fixed continuum $l=0,1, \cdots, L_{i}$, the above constraints state that the corresponding basis function $\psi_{l}^{i}$ has mean value zero on all continua within $K_{i}^{+}$and has mean value one on the fixed continuum.

In Fig. 3, we present pressure multiscale basis functions for matrix and fracture for some local domain with 3 oversampling layers. In this figure, the coarse grid is shown. The oversampling region is the larger square region marked red and the target coarse element is the smaller square marked red. We note that the oversampling region is defined as the union of $7 \times 7$ coarse elements.

The local problem we will solve is the original problem, but restricted to the oversampled domain $K_{i}^{+}$. In matrix form, the local problem can be written as

$$
\left(\begin{array}{cccc}
A_{m}^{K_{i}^{+}} & A_{m f}^{K_{i}^{+}} & B_{m}^{T} & 0 \\
A_{f m}^{K_{i}^{+}} & A_{f}^{K_{i}^{+}} & 0 & B_{f}^{T} \\
B_{m} & 0 & 0 & 0 \\
0 & B_{f} & 0 & 0
\end{array}\right)\left(\begin{array}{c}
\psi_{m} \\
\psi_{f} \\
\mu_{m} \\
\mu_{f}
\end{array}\right)=\left(\begin{array}{c}
0 \\
0 \\
F_{m} \\
F_{f}
\end{array}\right) .
$$

In Eq. 9, the solution is denoted by $\psi=\left(\psi_{m}, \psi_{f}\right)$, where $\psi_{m}$ and $\psi_{f}$ represent the solution on the matrix part and the fracture part, respectively, which is solved on the fine grid. The matrix $A_{m}^{K_{i}^{+}}$is the restriction of the matrix $A_{m}$ to the degrees of freedoms in the interior of the oversampling 
Fig. 3 Illustration of a $2 \mathrm{D}$ coarse-grid $\mathcal{T}_{H}(40 \times 40)$ and multiscale basis functions in local domain $K^{+}$with three oversampling layers

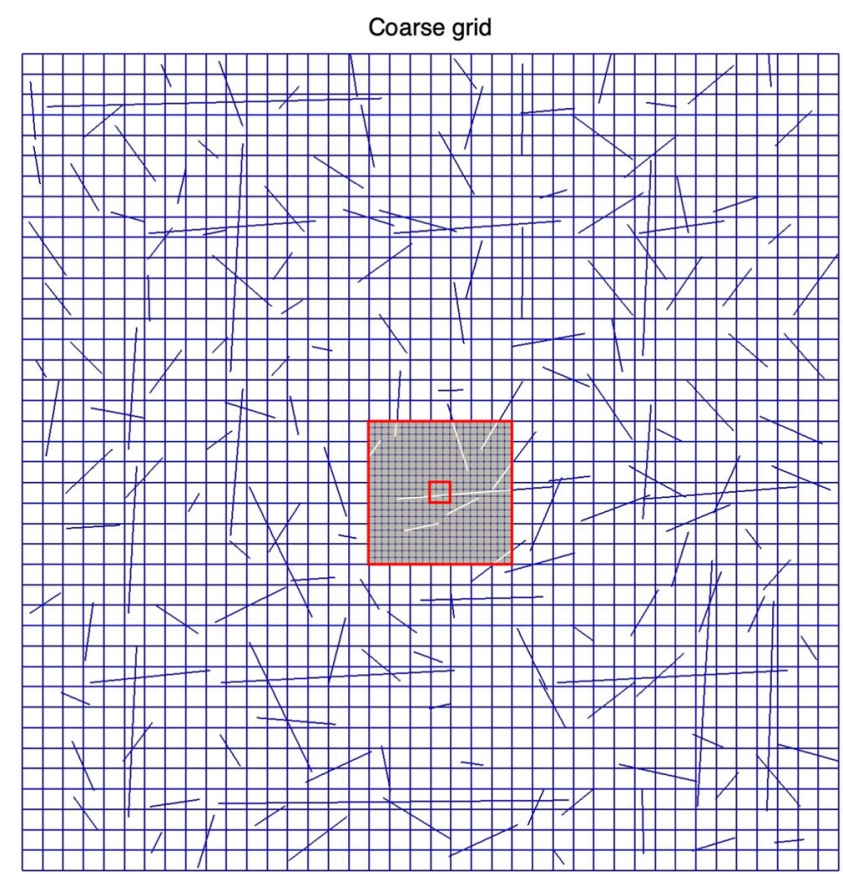

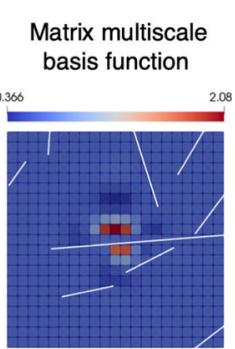

Fracture multiscale basis function

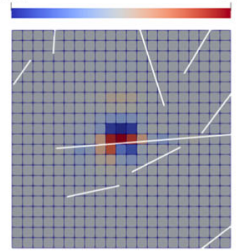

region $K_{i}^{+}$. The definitions for the matrices $A_{m f}^{K_{i}^{+}}, A_{f m}^{K_{i}^{+}}$ and $A_{f}^{K_{i}^{+}}$are similar. We remark that the zero Dirichlet boundary condition is imposed on the boundary of $K_{i}^{+}$. Furthermore, the variable $\mu=\left(\mu_{m}, \mu_{f}\right)$ plays the role of Lagrange multipliers for the constraints in Eqs. 7 and 8. The matrix $B_{m}$ corresponds to the mean value operators defined on matrix part and the matrix $B_{f}$ corresponds to the mean value operators defined on fracture networks. The righthand side vectors $F_{m}$ and $F_{f}$ are defined by the right-hand sides of Eqs. 7 and 8. We remark that Eq. 9 is the resulting variational form of an energy minimization idea originated from Chung et al. [12].

We write the basis functions as $\psi_{l}^{i}=\left(\psi_{m, l}^{i}, \psi_{f, l}^{i}\right)$, $l=0,1, \cdots, L_{i}$, where the subscripts $m$ and $f$ denote the matrix and the fracture parts, respectively. Combining these multiscale basis functions, we obtain the following multiscale space

$V_{\mathrm{ms}}=\operatorname{span}\left\{\psi_{l}^{i}=\left(\psi_{m, l}^{i}, \psi_{f, l}^{i}\right), \quad i=\overline{1, N_{c}}, \quad l=\overline{0, L_{i}}\right\}$

and the projection operator

$R=\left(\begin{array}{cc}R_{m} & R_{m f} \\ R_{f m} & R_{f}\end{array}\right)$,

where

$$
\begin{aligned}
& R_{m}^{T}=\left[\psi_{m, 0}^{0}, \psi_{m, 0}^{1} \ldots \psi_{m, 0}^{N_{c}}\right], \\
& R_{f}^{T}=\left[\psi_{f, 1}^{0} \ldots \psi_{f, L_{0}}^{0}, \psi_{f, 1}^{1} \ldots \psi_{f, L_{1}}^{1}, \ldots, \psi_{f, 1}^{N_{c}} \ldots \psi_{f, L_{N_{c}}}^{N_{c}}\right], \\
& R_{m f}^{T}=\left[\psi_{f, 0}^{0}, \psi_{f, 0}^{1} \ldots \psi_{f, 0}^{N_{c}}\right], \\
& R_{f m}^{T}=\left[\psi_{m, 1}^{0} \ldots \psi_{m, L_{0}}^{0}, \psi_{m, 1}^{1} \ldots \psi_{m, L_{1}}^{1}, \ldots, \psi_{m, 1}^{N_{c}} \ldots \psi_{m, L_{N_{c}}}^{N_{c}}\right] .
\end{aligned}
$$

We notice that the matrix $R$ gives a projection from the finescale space to the multiscale space $V_{\mathrm{ms}}$ and the matrix $R^{T}$ provides the mapping from the multiscale space back to the fine-scale space. In the above definition of the matrices, we assume that each multiscale basis function is represented in terms of the fine-scale basis and the coefficients of expansion are put as column vectors.

Using the local multiscale basis functions, we can define the required upscaled coarse-grid approximation as follows

$\bar{M} \frac{\bar{u}^{n+1}-\bar{u}^{n}}{\tau}+\bar{A} \bar{u}^{n+1}=\bar{F}$,

where

- $\quad$ for the pressure equation, $\bar{u}=\bar{p}$ :

$\bar{M}=R M^{\text {flow }} R^{T}, \quad \bar{A}=R A^{\text {flow }} R^{T}, \quad \bar{F}=R\left(G^{\text {flow }}+S^{\text {flow }} \frac{T-\check{T}}{\tau}\right)$.

- $\quad$ for the temperature equation, $\bar{u}=\bar{T}$ :

$$
\bar{M}=\mathcal{I} M^{\text {heat }} R^{T}, \quad \bar{A}=\mathcal{I} A^{\text {heat }} R^{T}, \quad \bar{F}=R\left(G^{\text {heat }}+S^{\text {heat }} \frac{T-\check{T}}{\tau}\right) .
$$

We note that $\bar{u}=\left(\bar{u}_{m}, \bar{u}_{f}\right)$ and $\bar{u}_{m}$ and $\bar{u}_{f}$ are the average cell solution on coarse-grid element for porous matrix and for fracture. We can reconstruct the downscale solution on the fine grid using the projection matrix $u=R^{T} \bar{u}$.

In the above definitions of the coarse-scale matrices, the operator $\mathcal{I}$ is the restriction operator

$\mathcal{I}=\left(\begin{array}{cc}\mathcal{I}_{m} & 0 \\ 0 & \mathcal{I}_{f}\end{array}\right)$ 

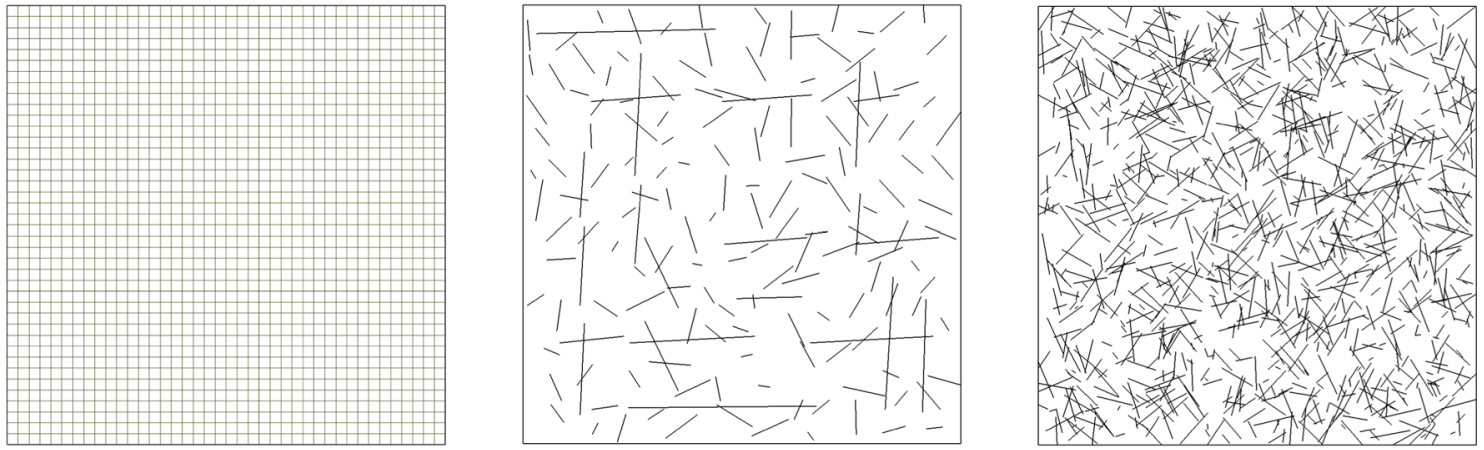

Fig. 4 Coarse mesh and fracture distributions. From left to right: $40 \times 40$ coarse mesh, Geometry 1 with 170 fracture lines, and Geometry 2 with 1000 fracture lines. Fine grid is $120 \times 120$ mesh with quadratic

which is defined by

$\mathcal{I}_{m}=\left\{\mathcal{I}_{i j}^{m}\right\}, \quad \mathcal{I}_{i j}^{m}=\left\{\begin{array}{cc}1, & \xi_{j} \in K_{i}, \\ 0 & \text { otherwise }\end{array}, \quad \mathcal{I}_{f}=\left\{\mathcal{I}_{l n}^{f}\right\}, \quad \mathcal{I}_{l n}^{f}=\left\{\begin{array}{cc}1, & \iota_{n} \in \gamma_{l}^{i}, \\ 0 & \text { otherwise }\end{array}\right.\right.$

Lastly, we will use the following definitions for the mass matrices and the right-hand side vectors:

$\bar{M}=\left(\begin{array}{cc}\bar{M}_{m} & 0 \\ 0 & \bar{M}_{f}\end{array}\right), \quad \bar{F}=\left(\begin{array}{c}\bar{F}_{m} \\ \bar{F}_{f}\end{array}\right)$,

where

$\bar{M}_{m}^{\text {flow }}=\operatorname{diag}\left\{a_{m}\left|K_{i}\right|\right\}, \quad \bar{M}_{f}^{\text {flow }}=\operatorname{diag}\left\{a_{f}\left|\gamma_{l}\right|\right\}$,

$\bar{M}_{m}^{\text {heat }}=\operatorname{diag}\left\{(c \rho)_{m}\left|K_{i}\right|\right\}, \quad \bar{M}_{f}^{\text {heat }}=\operatorname{diag}\left\{(c \rho)_{f}\left|\gamma_{l}\right|\right\}$,

and for the right-hand side vector $\bar{F}_{m}^{\alpha}=\left\{f_{m}^{\alpha}\left|K_{i}\right|\right\}, \bar{F}_{f}^{\alpha}=$ $\left\{f_{f}^{\alpha}\left|\gamma_{l}\right|\right\}$ for $\alpha=$ heat or flow.

In NLMC method, we have offline and online stages. In the offline stage: (1) we calculate multiscale basis functions; and (2) construct projection and prolongation operators. In the online stage: (1) we load the projection and prolongation operators; and (2) construct and solve the coarse-grid system; Therefore, for the heat and mass transfer problem, we have following computational algorithm for each time step:

- Solve pressure equation, $\bar{p}$,

- Update pressure dependent properties,

- Solve temperature equation, $\bar{T}$,

- Update temperature dependent properties.

Here, we took nonlinear coefficients from the previous time step, in general, iterative nonlinear solvers should be used. The main computational advantage of the multiscale approach is that the solution of the coarse-grid system is faster than solving the fine-grid system.

The implementation is constructed using $\mathrm{C}++$ programming language and is based on the open-source library FEniCS, where we use geometry objects and the FEniCS cells. The fine grid for fracture domain for Geometry 1 and Geometry 2 contains 3285 cells and 12,535 cells

interface to the linear solvers $[31,32]$. We use a direct solver for solution of the linear system of equations; however, an iterative method could be used with an appropriate preconditioner for this type of problems. Furthermore, the constructed projection and prolongation matrices can be used in the iterative multiscale solver.

\section{Numerical results}

In this section, we present several numerical results for the flow and heat transfer problem in two-dimensional fractured domain $\Omega=[0,1] \times[0,1] \mathrm{m}^{2}$, and $h$ the domain thickness is set to $1 \mathrm{~m}$.

We consider two test problems with two types of geometries:

- Geometry 1 with 170 fracture lines.

- Geometry 2 with 1000 fracture lines.

The coarse and fine grids are uniform with rectangular cells. In Fig. 4, the computational coarse grid with 1600 cells, fracture distribution for Geometry 1 and Geometry 2, is shown. Fine grid is $120 \times 120$ mesh with quadratic cells. We use $\mathrm{DoF}_{c}$ and $\mathrm{DoF}_{f}$ to denote, respectively, the problem size of the upscaled model and the fine-grid system:

- Geometry 1. $\mathrm{DoF}_{f}=17,685$ and $\mathrm{DoF}_{c}=2437$.

- Geometry 2. $\operatorname{DoF}_{f}=26,935$ and $\operatorname{DoF}_{c}=5104$.

The fine grid for fractures domain for Geometry 1 and Geometry 2 contain 3285 cells and 12,535 cells, respectively.

We consider following forms of Eqs. 2 and 3 scaled with respect to $p^{*}=10^{7} \mathrm{~Pa}$

$$
\begin{aligned}
& \tilde{a}_{m} \frac{\partial \tilde{p}_{m}}{\partial t}-s_{m} \frac{\partial T_{m}}{\partial t}-\operatorname{div}\left(\tilde{k}_{m} \operatorname{grad} \tilde{p}_{m}\right)+\tilde{\sigma} \eta_{m}\left(\tilde{p}_{m}-\tilde{p}_{f}\right)=g_{m}^{p}, \quad x \in \Omega, \\
& \tilde{a}_{f} \frac{\partial \tilde{p}_{f}}{\partial t}-s_{f} \frac{\partial T_{f}}{\partial t}-\operatorname{div}\left(\tilde{k}_{f} \operatorname{grad} \tilde{p}_{f}\right)-\tilde{\sigma} \eta_{f}\left(\tilde{p}_{m}-\tilde{p}_{f}\right)=g_{f}^{p}, \quad x \in \gamma,
\end{aligned}
$$



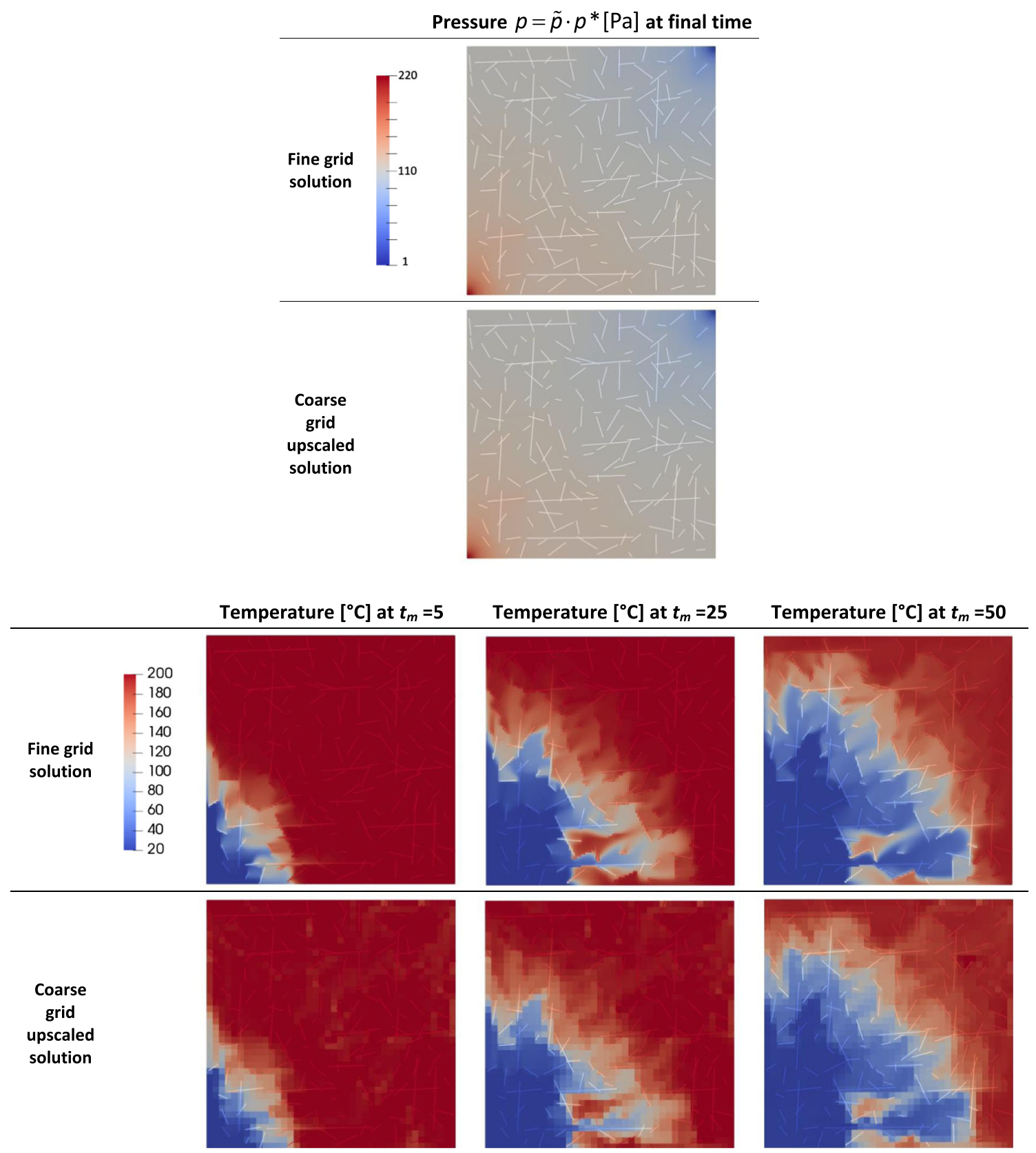

Fig. 5 Numerical results distribution for reference fine-grid solution and coarse-grid upscaled solution for three time steps $t_{m}, m=5,25$, and 50 . Geometry 1 with 170 fracture lines

and

$$
\begin{array}{rlr}
(c \rho)_{m} \frac{\partial T_{m}}{\partial t} & +(c \rho)_{w} \operatorname{div}\left(q_{m} T_{m}\right)+(c \rho)_{w} \tilde{\sigma} \eta_{m}\left(\tilde{p}_{m}-\tilde{p}_{f}\right) T_{m f} \\
& -\operatorname{div}\left(\lambda_{m} \operatorname{grad} T_{m}\right)+\beta \eta_{m}\left(T_{m}-T_{f}\right)=(c \rho)_{w} g_{m}^{T}, \quad x \in \Omega, \\
(c \rho)_{f} \frac{\partial T_{f}}{\partial t} & +(c \rho)_{w} \operatorname{div}\left(q_{f} T_{f}\right)+(c \rho)_{w} \tilde{\sigma} \eta_{f}\left(\tilde{p}_{m}-\tilde{p}_{f}\right) T_{m f} & \\
& -\operatorname{div}\left(\lambda_{f} \operatorname{grad} T_{f}\right)-\beta \eta_{f}\left(T_{m}-T_{f}\right)=(c \rho)_{w} g_{f}^{T}, \quad x \in \gamma,
\end{array}
$$

where $\tilde{a}_{n}=a_{n} \cdot p^{*}, \tilde{k}_{n}=k_{n} \cdot p^{*}, \tilde{\sigma}=\sigma \cdot p^{*}, \tilde{p}_{n}=p_{n} / p^{*}$ for $n=m, f$ and $p^{*}=10^{7} \mathrm{~Pa}$.
We set the following parameters:

- the pressure equation:

$$
a_{m}=a_{f}=10^{-6}[1 / \mathrm{Pa}]\left(\tilde{a}_{m}=\tilde{a}_{f}=10\right), c_{T}=0
$$$$
\left(s_{m}=s_{f}=0\right), k_{m}=10^{-13}, k_{f}=10^{-7}\left[\mathrm{~m}^{2} / \mathrm{Pa} . \mathrm{s}\right] \text {, }
$$$$
\left(\tilde{k}_{m}=10^{-6}, \tilde{k}_{f}=1\right) \text {, and } \tilde{\sigma}=C I \cdot \tilde{k}_{m} \text {, }
$$

- the temperature equation:

$$
\lambda_{m}=0.1, \lambda_{f}=1\left[\mathrm{~W} /{ }^{\circ} \mathrm{C} . \mathrm{m}\right],(c \rho)_{m}=(c \rho)_{f}=
$$$$
(c \rho)_{w}=10^{6}\left[\mathrm{~J} /{ }^{\circ} \mathrm{C} \cdot \mathrm{m}^{3}\right], c_{T}=0 \text {, and } \beta=C I \cdot \lambda_{m} \text {. }
$$

We set solution of the steady-state problem for pressure as initial condition and zero flux on boundaries for pressure 
Fig. 6 Numerical results distribution for reference fine-grid solution (the first row), coarse-grid upscaled solution (the second row), and reconstructed fine-grid solution (the third row) for three time steps $t_{m}, m=5,25$, and 50 . Geometry 2 with 1000 fracture lines

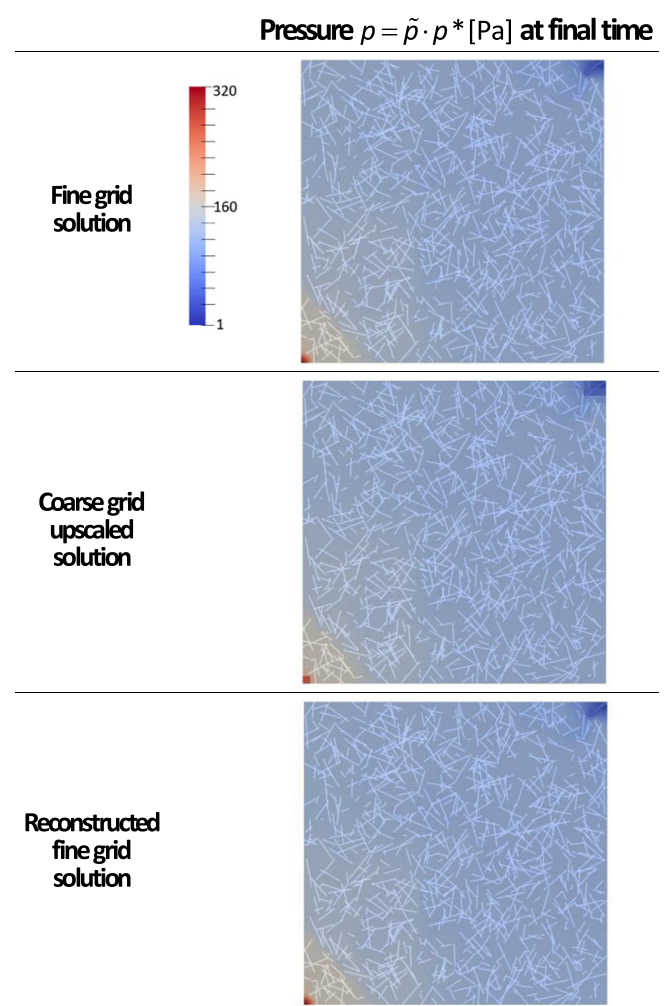

Temperature $\left({ }^{\circ} \mathrm{C}\right)$ at $t_{m}=5$

Temperature $\left({ }^{\circ} \mathrm{C}\right)$ at $t_{m}=25$

Temperature $\left({ }^{\circ} \mathrm{C}\right)$ at $t_{m}=50$

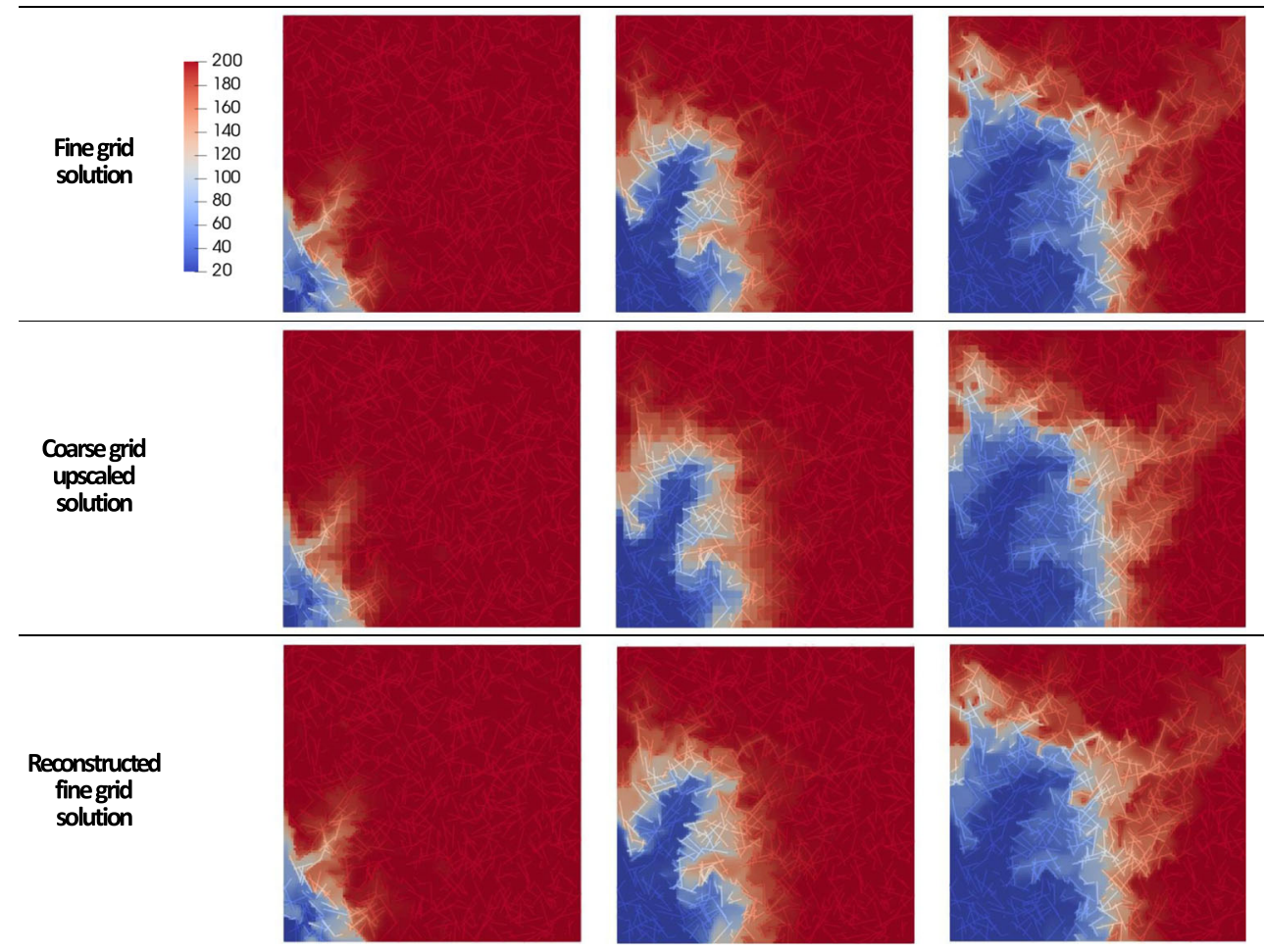

and temperature. Initial condition for temperature is $T_{0}=$ $200\left[{ }^{\circ} \mathrm{C}\right]$ and we inject cold fluid with temperature $T_{1}=20$ $\left[{ }^{\circ} \mathrm{C}\right]$. We set a source term on the cells $\Omega_{\text {inj }}=[0.0-$ $0.025] \mathrm{m} \times[0.0-0.025] \mathrm{m}$ and $\Omega_{\mathrm{prd}}=[0.975-1.0]$ $\mathrm{m} \times[0.975-1.0] \mathrm{m}$ with $g_{m}^{p}=1[1 / \mathrm{sec}]$ for $\Omega_{\mathrm{inj}}, g_{m}^{p}=-1$ [1/sec] for $\Omega_{\text {prd }}$ and $g_{f}^{p}=0$. For the temperature equation source/sink terms: $g_{m, f}^{T}=T_{1} g_{m, f}^{p}$ for $\Omega_{\text {inj }}$, and $g_{m, f}^{T}=$ $T_{m, f} g_{m, f}^{p}$ for $\Omega_{\mathrm{prd}, m, f}$, where $T_{\Omega_{\mathrm{prd}, m, f}}$ is the matrix or 
Table 1 Numerical results of relative errors (\%) for heat (left table) and flow (right table) at the final time

\begin{tabular}{llllll}
\hline$K^{M}$ & $e_{\text {heat }}^{C}$ & $e_{\text {heat }}^{F}$ & $K^{M}$ & $e_{\text {flow }}^{C}$ & $e_{\text {flow }}^{F}$ \\
\hline Geometry 1 & & & & & \\
1 & - & - & 1 & 0.183 & 14.419 \\
2 & 46.370 & 60.557 & 2 & 0.258 & 3.082 \\
3 & 25.879 & 33.197 & 3 & 0.333 & 0.664 \\
4 & 8.109 & 10.568 & 4 & 0.360 & 0.380 \\
6 & 1.222 & 1.629 & 6 & 0.357 & 0.361 \\
& & & & & \\
Geometry 2 & & & & & - \\
1 & - & - & 2 & 0.134 & 2.066 \\
2 & 43.955 & 60.192 & 3 & 0.034 & 0.206 \\
3 & 22.208 & 33.411 & 4 & 0.031 & 0.041 \\
4 & 1.257 & 1.717 & 5 & 0.031 & 0.031 \\
6 & 0.088 & 0.113 & & & \\
\hline
\end{tabular}

Coarse grid $40 \times 40$ is used. Geometry 1 and 2 fracture temperature of production region. Simulation time is set to $t_{\max }=10,000 \mathrm{~s}$ for Geometry 1 and $t_{\max }=$ $30,000 \mathrm{~s}$ for Geometry 2 both with 50 time steps.

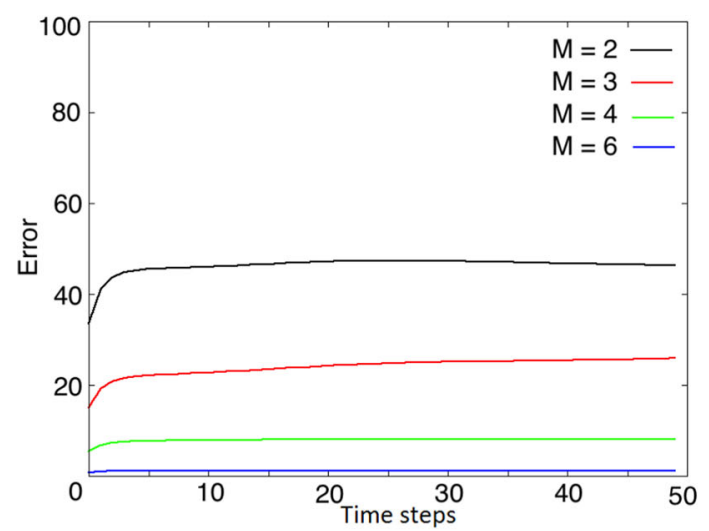

Relative errors $\left(e_{\text {heat }}^{c}\right)$ vs. time for Geometry 1

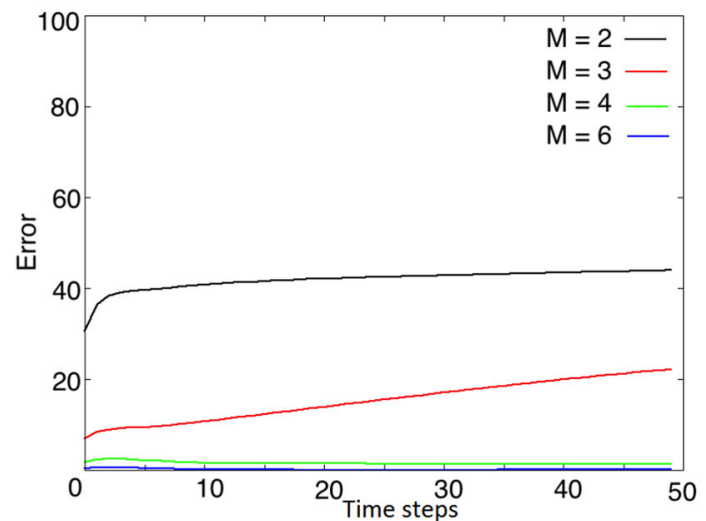

Relative errors $\left(e_{\text {heat }}^{c}\right)$ vs. time for Geometry 2
In Fig. 5, we present the fine-scale and upscaled solutions for Geometry 1 and in Fig. 6 for Geometry 2 at different time steps $t_{m}$ with $m=5,25$, and 50, corresponding to

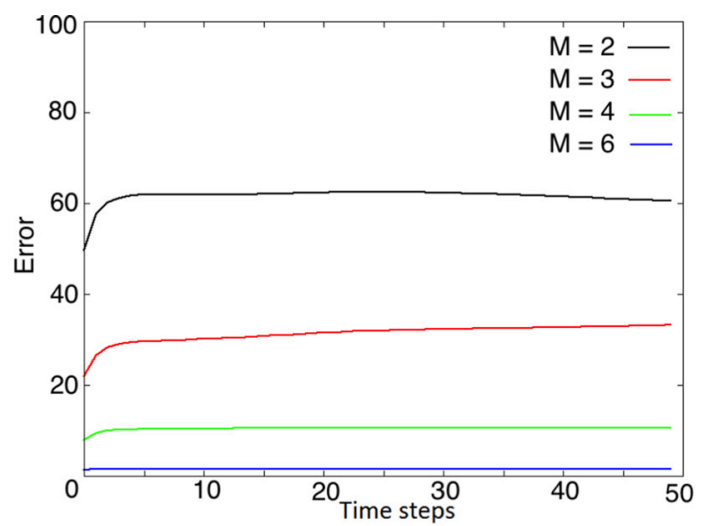

Relative errors $\left(e_{\text {heat }}^{F}\right)$ vs. time for Geometry 1

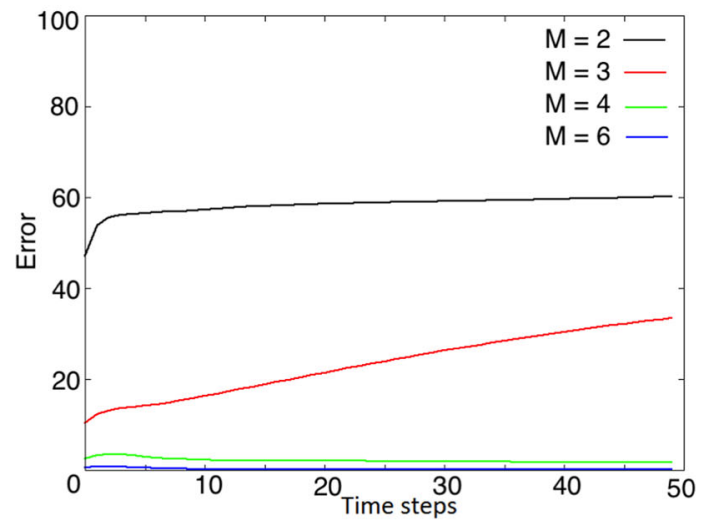

Relative errors $\left(e_{\text {heat }}^{F}\right)$ vs. time for Geometry 2

Fig. 7 Relative errors for temperature vs. time with different number of oversampling layers 


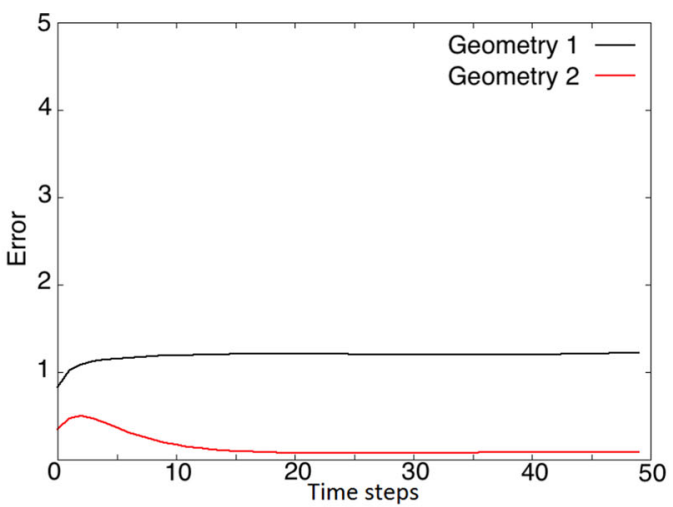

Relative errors $\left(e_{\text {heat }}^{c}\right)$ vs. time for Geometry 1 and 2 $(M=6)$

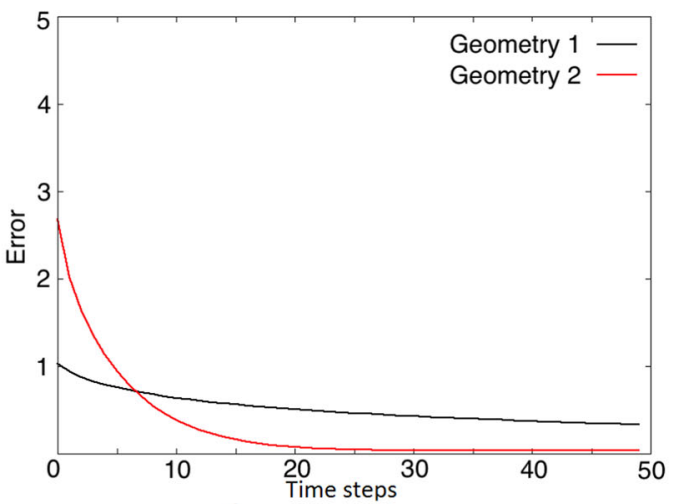

Relative errors $\left(e_{\text {flow }}^{c}\right)$ vs. time for Geometry 1 and 2 $(M=3)$

Fig. 8 Relative errors vs. time for temperature and pressure

$t=1000,5000$, and $10,000 \mathrm{~s}$ for Geometry 1 , and $t=$ $3000,15,000$, and 30,000 s for Geometry 2. The first row presents solutions on the fine grid. The second row shows the coarse-grid upscaled solution. The pressure is shown at the final time. For basis calculations, we use oversampled domain $K^{M}$, with 6 oversampling layers for temperature and 3 oversampling layers for pressure. We observe a good agreement between the fine-scale solution and the computed upscaled solution for Geometry 1 and Geometry 2.

To quantitatively compare the results, we use the relative $L^{2}$ error between reference fine-grid solution and upscaled coarse-grid solution. We calculate errors on coarse grid $\left(e^{C}\right)$ and on fine grid $\left(e^{F}\right)$ :

$e_{\alpha}^{C}=\frac{\left\|u_{C}-\bar{u}\right\|_{L^{2}}}{\left\|u_{C}\right\|_{L^{2}}}, \quad e_{\alpha}^{F}=\frac{\left\|u-\bar{u}_{F}\right\|_{L^{2}}}{\|u\|_{L^{2}}}$,

where $u=T$ for $\alpha=$ heat, and $u=p$ for $\alpha=$ flow, $\bar{u}$ is the upscaled coarse-grid solution, $\bar{u}_{F}=R^{T} \bar{u}$ is the reconstructed fine-grid solution from $\bar{u}, u$ is the reference

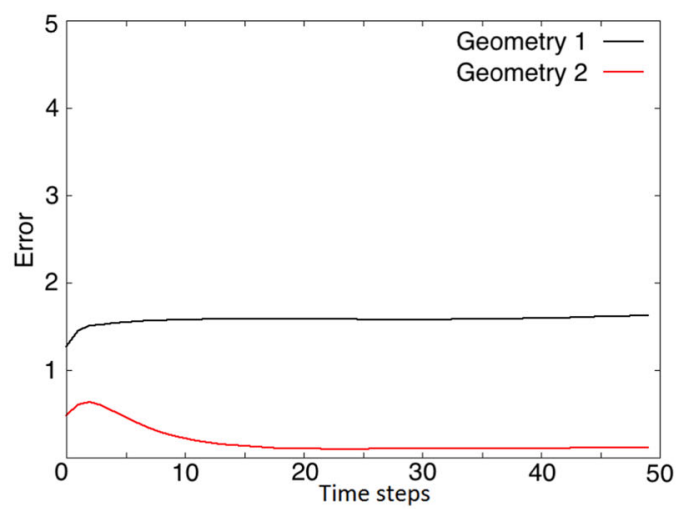

Relative errors $\left(e_{\text {heat }}^{F}\right)$ vs. time for Geometry 1 and 2

$(M=6)$

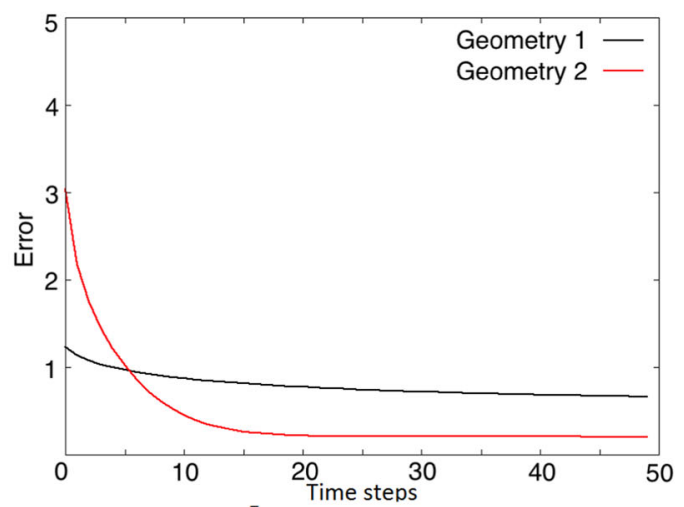

Relative errors $\left(e_{\text {flow }}^{F}\right)$ vs. time for Geometry 1 and 2

$(M=3)$

fine-grid solution, $u_{C}$ is the coarse-grid cell average for reference fine-grid solution $u$, and

$\left\|u_{C}-\bar{u}\right\|_{L^{2}}^{2}=\frac{1}{N_{c}} \sum_{i=1}^{N_{c}}\left(u_{C}^{K_{i}}-\bar{u}^{K_{i}}\right)^{2}, \quad u_{C}^{K_{i}}=\frac{1}{\left|K_{i}\right|} \int_{K_{i}} u d x$.

In Table 1, we present relative errors in heat (temperature) and flow (pressure) for two geometries and different number of oversampling layers $K^{M}$ with $M=1,2,3,4$, and 6 . We observe a good convergence behavior when a sufficient number of oversampled layers is used. For example, there is less than a $1.2 \%$ of relative error for temperature using 6 oversampling layers for Geometry 1 and $0.09 \%$ for Geometry 2 at the final time. It is evident that for flow problem, a smaller number of oversampling layers can be used, compared to heat problem, to obtain small error margins.

Finally, Figs. 7 and 8 demonstrate the temporal evolution of error. Very small error is observed for the final times of simulations using $M=6$. The error of flow is much smaller than the error of temperature because of the fact 
that the pressure equation is a parabolic equation, and the proposed method works extremely well in this case. For temperature, the convection term is a challenging part, and the performance of the method can be reduced. Furthermore, the rapid decay of error for Geometry 2 compared to Geometry 1 can be attributed to the fracture density and distribution. Although Geometry 1 has sparser fracture density but it has longer range fractures that span larger extent across the domain than those of Geometry 2. The longer range extent of fractures in Geometry 1 is responsible for slower decay of error compared to Geometry 2.

\section{Conclusions and future works}

The application of a new multiscale method for coupled heat and flow problem showed a good agreement between the fine-grid solution and upscaled coarse-grid solution.

In the current work, we assumed a small diffusion dominated heat transfer processes. However, the diffusion process may impact the hydrodynamics of mass exchange between fractures and matrix. In future, we will add mechanical effects and consider coupled thermoporoelastic processes in fractured media. Such numerical applications have been discussed in existing literature studies [52], but not in a computationally efficient multiscale framework. Moreover, in the context of coupled thermo-hydromechanical-chemical (THMC) processes, fracture alteration is an area of research for the geothermal heat recovery [6, $7,36,41,42]$. Computational expenses are always an issue even for modern computers. In future, we explore extending multiscale modeling to these areas of research as well.

Acknowledgements The authors thank the anonymous reviewers for their constructive comments.
Funding MV and VA's works are supported by the grant of Russian Scientific Fund N17-71-20055 and the mega-grant of Russian Federation Government (N 14.Y26.31.0013). MB's contribution was made through CUHK-UoM Research Fund. EC and MB's work are also partially supported by Hong Kong RGC General Research Fund (Project 14304217), CUHK Direct Grant for Research 2017-18, and CUHK-UoM Research Fund.

Open Access This article is distributed under the terms of the Creative Commons Attribution 4.0 International License (http:// creativecommons.org/licenses/by/4.0/), which permits unrestricted use, distribution, and reproduction in any medium, provided you give appropriate credit to the original author(s) and the source, provide a link to the Creative Commons license, and indicate if changes were made.

\section{Appendix}

In this Appendix, we present the detailed definitions of the fine-scale matrices introduced in Section 3. Our solution strategy includes:

- Solve the pressure equation and find $p=\left(p_{m}, p_{f}\right)^{T}$

$$
M^{\text {flow }} \frac{p-\check{p}}{\tau}+A^{\text {flow }} p=G^{\text {flow }}+S^{\text {flow }} \frac{T-\check{T}}{\tau},
$$

where

$$
\begin{gathered}
M^{\text {flow }}=\left(\begin{array}{cc}
M_{m}^{\text {flow }} & 0 \\
0 & M_{f}^{\text {flow }}
\end{array}\right), \quad S^{\text {flow }}=\left(\begin{array}{cc}
S_{m}^{\text {flow }} & 0 \\
0 & S_{f}^{\text {flow }}
\end{array}\right), \quad G^{\text {flow }}=\left(\begin{array}{c}
G_{m}^{\text {flow }} \\
G_{f}^{\text {flow }}
\end{array}\right), \\
A^{\text {flow }}=\left(\begin{array}{cc}
A_{m}^{\text {flow }}+Q & -Q \\
-Q & A_{f}^{\text {flow }}+Q
\end{array}\right),
\end{gathered}
$$

- Solve the temperature equation and find $T=\left(T_{m}, T_{f}\right)^{T}$

$$
M^{\text {heat }} \frac{T-\check{T}}{\tau}+A^{\text {heat }}(p) T=G^{\text {heat }}+S^{\text {heat }} \frac{p-\check{p}}{\tau} \text {, }
$$

where

$$
\begin{aligned}
& M^{\text {heat }}=\left(\begin{array}{cc}
M_{m}^{\text {heat }} & 0 \\
0 & M_{f}^{\text {heat }}
\end{array}\right), \quad S^{\text {heat }}=\left(\begin{array}{cc}
S_{m}^{\text {heat }} & 0 \\
0 & S_{f}^{\text {heat }}
\end{array}\right), \quad G^{\text {heat }}=\left(\begin{array}{c}
G_{m}^{\text {heat }} \\
G_{f}^{\text {heat }}
\end{array}\right), \\
& A^{\text {heat }}=\left(\begin{array}{cc}
A_{m}^{\text {heat }}+C_{m}^{U P}\left(p_{m}\right)+L^{U P}\left(p_{m}, p_{f}\right)+\Theta & -\Theta+L^{U P}\left(p_{m}, p_{f}\right) \\
-\Theta-L^{U P}\left(p_{m}, p_{f}\right) & A_{f}^{\text {heat }}+C_{f}^{U P}\left(p_{f}\right)-L^{U P}\left(p_{m}, p_{f}\right)+\Theta
\end{array}\right),
\end{aligned}
$$

where

$$
\begin{aligned}
& M_{m}^{\text {flow }}=\left\{m_{i j}^{\text {flow }, m}\right\}, \quad m_{i j}^{\text {flow }, m}=\left\{\begin{array}{cc}
a_{m, i}\left|\xi_{i}\right| & i=j, \\
0 & i \neq j
\end{array},\right. \\
& M_{f}^{\text {flow }}=\left\{m_{l n}^{\text {flow }, f}\right\}, \quad m_{l n}^{\text {flow }, f}=\left\{\begin{array}{cc}
a_{f, l}\left|\iota_{l}\right| & l=n, \\
0 & l \neq n
\end{array},\right. \\
& M_{m}^{\text {heat }}=\left\{m_{i j}^{\text {heat }, m}\right\}, \quad m_{i j}^{\text {heat }, m}=\left\{\begin{array}{cc}
(c \rho)_{m, i}\left|\xi_{i}\right| & i=j, \\
0 & i \neq j
\end{array},\right.
\end{aligned}
$$

$$
\begin{aligned}
& M_{f}^{\text {heat }}=\left\{m_{l n}^{\text {heat, } f}\right\}, \quad m_{l n}^{\text {heat, } f}=\left\{\begin{array}{cc}
(c \rho)_{f, l}\left|\iota_{l}\right| & l=n, \\
0 & l \neq n
\end{array},\right. \\
& S_{m}^{\text {flow }}=\left\{s_{i j}^{\text {flow }, m}\right\}, \quad s_{i j}^{\text {flow }, m}=\left\{\begin{array}{cc}
s_{m, i}\left|\xi_{i}\right| & i=j, \\
0 & i \neq j
\end{array},\right. \\
& S_{f}^{\text {flow }}=\left\{s_{l n}^{\text {flow }, f}\right\}, \quad s_{l n}^{\text {flow, } f}=\left\{\begin{array}{cc}
s_{f, l}\left|\iota_{l}\right| & l=n, \\
0 & l \neq n
\end{array},\right. \\
& S_{m}^{\text {heat }}=\left\{s_{i j}^{\text {heat }, m}\right\}, \quad s_{i j}^{\text {heat }, m}=\left\{\begin{array}{cc}
c_{T, i} \check{T}_{m, i}\left|\xi_{i}\right| & i=j, \\
0 & i \neq j
\end{array},\right.
\end{aligned}
$$




$$
\begin{aligned}
& S_{f}^{\text {heat }}=\left\{s_{l n}^{\text {heat, } f}\right\}, \quad s_{l n}^{\text {heat }, f}=\left\{\begin{array}{cc}
c_{T, l} \check{T}_{f, l}\left|\iota_{l}\right| & l=n, \\
0 & l \neq n
\end{array},\right. \\
& Q=\left\{q_{i l}\right\}, \quad q_{i l}=\left\{\begin{array}{cc}
\sigma & i=l, \\
0 & i \neq l
\end{array}, \quad \Theta=\left\{\theta_{i l}\right\}, \quad \theta_{i l}=\left\{\begin{array}{cc}
\beta & i=l, \\
0 & i \neq l
\end{array}\right.\right. \\
& \text { and } A_{m}^{\text {flow }}=\left\{W_{i j}^{m}\right\}, A_{f}^{\text {flow }}=\left\{W_{l n}^{f}\right\}, A_{m}^{\text {heat }}=\left\{D_{i j}^{m}\right\} \text {, } \\
& A_{f}^{\text {heat }}=\left\{D_{l n}^{f}\right\}, G_{m}^{\alpha}=\left\{g_{m, i}^{\alpha}\left|\xi_{i}\right|\right\}, G_{f}^{\alpha}=\left\{g_{f, l}^{\alpha}\left|\iota_{l}\right|\right\} \text {, where } \\
& \alpha=\text { heat or flow. For convection terms } C_{m}^{U P} \text { and } \\
& C_{f}^{U P} \text {, we use upwind scheme and } L^{U P} \text { is the convective } \\
& \text { mass transfer between matrix and fracture with upwind } \\
& \text { approximation. }
\end{aligned}
$$

\section{References}

1. Akkutlu, I.Y., Efendiev, Y., Vasilyeva, M.: Multiscale model reduction for shale gas transport in fractured media. Comput. Geosci., 1-21 (2015)

2. Akkutlu, I.Y., Efendiev, Y., Vasilyeva, M., Wang, Y.: Multiscale model reduction for shale gas transport in poroelastic fractured media. J. Comput. Phys. 353, 356-376 (2018)

3. Arbogast, T., Douglas, J.rJ., Hornung, U.: Derivation of the double porosity model of single phase flow via homogenization theory. SIAM J. Math. Anal. 21(4), 823-836 (1990)

4. Babaei, M.: Integrated carbon sequestration-geothermal heat recovery: performance comparison between open and close systems. Transp. Porous Media, 1-25 (2018)

5. Barenblatt, G.I., Zheltov, I.P., Kochina, I.N.: Basic concepts in the theory of seepage of homogeneous liquids in fissured rocks [strata]. J. Appl. Math. Mech. 24(5), 1286-1303 (1960)

6. Bisdom, K., Bertotti, G., Nick, H.M.: A geometrically based method for predicting stress-induced fracture aperture and flow in discrete fracture networks. AAPG Bull. 100(7), 1075-1097 (2016)

7. Bisdom, K., Nick, H.M., Bertotti, G.: An integrated workflow for stress and flow modelling using outcrop-derived discrete fracture networks. Comput. Geosci. 103, 21-35 (2017)

8. Bosma, S., Hajibeygi, H., Tene, M., Tchelepi, H.A.: Multiscale finite volume method for discrete fracture modeling on unstructured grids (MS-DFM). J. Comput. Phys. (2017)

9. Chung, E.T., Efendiev, Y., Li, G., Vasilyeva, M.: Generalized multiscale finite element method for problems in perforated heterogeneous domains. Appl. Anal. 255, 1-15 (2015)

10. Chung, E.T., Efendiev, Y., Hou, T.Y.: Adaptive multiscale model reduction with generalized multiscale finite element methods. J. Comput. Phys. 320, 69-95 (2016)

11. Chung, E.T., Efendiev, Y., Leung, T., Vasilyeva, M.: Coupling of multiscale and multi-continuum approaches. GEM-Int. J. Geomath. 8(1), 9-41 (2017)

12. Chung, E.T., Efendiev, Y., Leung, W.T.: Constraint energy minimizing generalized multiscale finite element method. Comput. Methods Appl. Mech. Eng. 339, 298-319 (2018)

13. Chung, E.T., Efendiev, Y., Leung, W.T., Vasilyeva, M., Wang, Y.: Non-local multi-continua upscaling for flows in heterogeneous fractured media. J. Comput. Phys. 372, 22-34 (2018)

14. Crooijmans, R.A., Willems, C.J.L., Nick, H.M., Bruhn, D.F.: The influence of facies heterogeneity on the doublet performance in low-enthalpy geothermal sedimentary reservoirs. Geothermics 64, 209-219 (2016)

15. D'Angelo, C., Scotti, A.: A mixed finite element method for Darcy flow in fractured porous media with non-matching grids. ESAIM: Math. Modell. Numer. Anal. 46(2), 465-489 (2012)
16. Efendiev, Y., Hou, T.: Multiscale Finite Element Methods: Theory and Applications, Surveys and Tutorials in the Applied Mathematical Sciences, vol. 4. Springer, New York (2009)

17. Efendiev, Y., Galvis, J., Hou, T.: Generalized multiscale finite element methods. J. Comput. Phys. 251, 116-135 (2013)

18. Efendiev, Y., Lee, S., Li, G., Yao, J., Zhang, N.: Hierarchical multiscale modeling for flows in fractured media using generalized multiscale finite element method. arXiv:150203828 (2015)

19. Fairley, J., Ingebritsen, S., Podgorney, R.: Challenges for numerical modeling of enhanced geothermal systems. Groundwater 48(4), 482 (2010)

20. Formaggia, L., Fumagalli, A., Scotti, A., Ruffo, P.: A reduced model for Darcy's problem in networks of fractures. ESAIM: Math. Modell. Numer. Anal. 48(4), 1089-1116 (2014)

21. Fumagalli, A., Pasquale, L., Zonca, S., Micheletti, S.: An upscaling procedure for fractured reservoirs with embedded grids. Water Resour. Res. 52(8), 6506-6525 (2016)

22. Gallup, D.L.: Production engineering in geothermal technology: a review. Geothermics 38(3), 326-334 (2009)

23. Ginting, V., Pereira, F., Presho, M., Wo, S.: Application of the two-stage Markov Chain Monte Carlo method for characterization of fractured reservoirs using a surrogate flow model. Comput. Geosci. 15(4), 691 (2011)

24. Hajibeygi, H., Kavounis, D., Jenny, P.: A hierarchical fracture model for the iterative multiscale finite volume method. J. Comput. Phys. 230(24), 8729-8743 (2011). https://doi.org/10.1016/j.jcp.2011.08. 021

25. Hao, Y., Fu, P., Johnson, S.M., Carrigan, C.R.: Numerical studies of coupled flow and heat transfer processes in hydraulically fractured geothermal reservoirs. In: Proceedings of the 36th Annual Meeting of Geothermal Resources Council Reno NV (2012)

26. He, Y.L., Tao, W.Q.: Multiscale simulations of heat transfer and fluid flow problems. J. Heat Transfer 134(3), 031018 (2012)

27. Hou, T., Wu, X.: A multiscale finite element method for elliptic problems in composite materials and porous media. J. Comput. Phys. 134, 169-189 (1997)

28. Jenny, P., Lee, S.H., Tchelepi, H.A.: Adaptive multiscale finitevolume method for multiphase flow and transport in porous media. Multiscale Model. Simul. 3(1), 50-64 (2005)

29. Karvounis, D.C., Jenny, P.: Adaptive hierarchical fracture model for enhanced geothermal systems. Multiscale Model. Simul. 14(1), 207-231 (2016)

30. Klepikova, M.V., Le Borgne, T., Bour, O., Dentz, M., Hochreutener, R., Lavenant, N.: Heat as a tracer for understanding transport processes in fractured media: theory and field assessment from multiscale thermal push-pull tracer tests. Water Resour. Res. 52(7), 5442-5457 (2016)

31. Logg, A.: Efficient representation of computational meshes. Int. J. Comput. Sci. Eng. 4(4), 283-295 (2009)

32. Logg, A., Mardal, K.A., Wells, G.: Automated solution of differential equations by the finite element method: the FEniCS book, vol 84. Springer Science \& Business Media (2012)

33. Lunati, I., Jenny, P.: Multiscale finite-volume method for compressible multiphase flow in porous media. J. Comput. Phys. 216(2), 616-636 (2006)

34. Martin, V., Jaffré, J., Roberts, J.E.: Modeling fractures and barriers as interfaces for flow in porous media. SIAM J. Sci. Comput. 26(5), 1667-1691 (2005)

35. Nissen, A., Keilegavlen, E., Sandve, T.H., Berre, I., Nordbotten, J.M.: Heterogeneity preserving upscaling for heat transport in fractured geothermal reservoirs. Comput. Geosci., 1-17 (2017)

36. Pandey, S., Chaudhuri, A., Kelkar, S.: A coupled thermo-hydromechanical modeling of fracture aperture alteration and reservoir deformation during heat extraction from a geothermal reservoir. Geothermics 65, 17-31 (2017) 
37. Pavlova, N.V., Vabishchevich, P.N., Vasilyeva, M.V.: Mathematical modeling of thermal stabilization of vertical wells on high performance computing systems. In: International Conference on Large-Scale Scientific Computing, pp. 636-643. Springer (2013)

38. Portier, S., Vuataz, F.D.: Developing the ability to model acid-rock interactions and mineral dissolution during the rma stimulation test performed at the Soultz-sous-Forêts EGS site, France. Compt. Rendus Geosci. 342(7-8), 668-675 (2010)

39. Praditia, T., Helmig, R., Hajibeygi, H.: Multiscale formulation for coupled flow-heat equations arising from single-phase flow in fractured geothermal reservoirs. Comput. Geosci., 1-18 (2018)

40. Rawal, C., Ghassemi, A.: A reactive thermo-poroelastic analysis of water injection into an enhanced geothermal reservoir. Geothermics 50, 10-23 (2014)

41. Salimzadeh, S., Nick, H.M., Zimmerman, R.: Thermoporoelastic effects during heat extraction from low-permeability reservoirs. Energy 142, 546-558 (2018)

42. Salimzadeh, S., Paluszny, A., Nick, H.M., Zimmerman, R.W.: A three-dimensional coupled thermo-hydro-mechanical model for deformable fractured geothermal systems. Geothermics 71, 212224 (2018)

43. Sandve, T.H., Berre, I., Keilegavlen, E., Nordbotten, J.M.: Multiscale simulation of flow and heat transport in fractured geothermal reservoirs: inexact solvers and improved transport upscaling. In: Thirty-Eighth Workshop on Geothermal Reservoir Engineering Stanford University, pp. 11-13 (2013)

44. Tambue, A., Berre, I., Nordbotten, J.M.: Efficient simulation of geothermal processes in heterogeneous porous media based on the exponential Rosenbrock-Euler and Rosenbrock-type methods. Adv. Water Resour. 53, 250-262 (2013)

45. Tene, M., Al Kobaisi, M.S., Hajibeygi, H.: Algebraic multiscale method for flow in heterogeneous porous media with embedded discrete fractures (F-AMS). J. Comput. Phys. 321, 819-845 (2016)

46. Tene, M., Bosma, S.B.M., Al Kobaisi, M.S., Hajibeygi, H.: Projection-based embedded discrete fracture model (PEDFM). Adv. Water Resour. 105, 205-216 (2017)

47. Tester, J.W., Anderson, B.J., Batchelor, A.S., Blackwell, D.D., DiPippo, R., Drake, E.M., Garnish, J., Livesay, B., Moore, M.C., Nichols, K., et al: Impact of enhanced geothermal systems on us energy supply in the twenty-first century. Philos. Trans. R. Soc. London A: Math. Phys. Eng. Sci. 365(1853), 1057-1094 (2007)

48. Vasilyeva, M., Babaei, M., Chung, E.T., Spiridonov, D.: Multiscale modelling of heat and mass transfer in fractured media for enhanced geothermal systems (EGS) applications. Appl. Math. Model. 67, 159-178 (2018)

49. Vasilyeva, M., Chung, E.T., Efendiev, Y., Kim, J.: Constrained energy minimization based upscaling for coupled flow and mechanics. arXiv:180509382 (2018)

50. Vasilyeva, M., Chung, E.T., Leung, W.T., Alekseev, V.: Nonlocal multicontinuum (nlmc) upscaling of mixed dimensional coupled flow problem for embedded and discrete fracture models. arXiv:180509407 (2018)

51. Vasilyeva, M., Chung, E.T., Leung, W.T., Wang, Y., Spiridonov, D.: Upscaling method for problems in perforated domains with non-homogeneous boundary conditions on perforations using non-local multi-continuum method (nlmc). arXiv:180509420 (2018)

52. Vik, H.S., Salimzadeh, S., Nick, H.M.: Heat recovery from multiple-fracture enhanced geothermal systems: the effect of thermoelastic fracture interactions. Renew. Energy 121, 606-622 (2018)

53. Wang, X., Ghassemi, A.: A 3d thermal-poroelastic model for geothermal reservoir stimulation. In: Thirty-Seventh Workshop on Geothermal Reservoir Engineering (2012)

54. Wang, X., Ghassemi, A.: A three-dimensional poroelastic model for naturally fractured geothermal reservoir stimulation In: GRC Annual Meeting. Las Vegas (2013)

55. Warren, J.E., Root, P.J.: The behavior of naturally fractured reservoirs. Soc. Pet. Eng. J. 3(03), 245-255 (1963)

56. Weinan, E., Engquist, B., Li, X., Ren, W., Vanden-Eijnden, E.: Heterogeneous multiscale methods: A review. Commun. Comput. Phys. 2(3), 367-450 (2007)

57. Willems, C.J.L., Nick, H.M., Weltje, G.J., Bruhn, D.F.: An evaluation of interferences in heat production from low enthalpy geothermal doublets systems. Energy 135, 500-512 (2017)

Publisher's note Springer Nature remains neutral with regard to jurisdictional claims in published maps and institutional affiliations. 